\title{
Acute kidney injury in moderate and severe COVID-19 patients: Report of two university hospitals
}

\author{
DANIELA RADULESCU ${ }^{1,2}$, LILIANA-ANA TUTA ${ }^{3,4}$, CRISTIANA DAVID $^{1,2}$, CARMEN BOGEANU $^{1,2}$, \\ SIMONA DANIELA ONOFREI ${ }^{1,2}$, ELENA STEPAN ${ }^{2}$, ELENA CUIBAN $^{2}$, ANDREEA CIOFALCA $^{2}$, \\ LARISA FLORINA FEIER ${ }^{2}$, CAMELIA PANA $^{3,4}$, MAGDA-CRISTINA NUTU $^{3,4}$ and ILEANA ADELA VACAROIU ${ }^{1,2}$ \\ ${ }^{1}$ Clinical Department No. 3, 'Carol Davila' University of Medicine and Pharmacy, 050474 Bucharest; \\ ${ }^{2}$ Department of Nephrology and Dialysis, 'Sf. Ioan' Emergency Clinical Hospital, 421422 Bucharest; \\ ${ }^{3}$ Faculty of Medicine, 'Ovidius' University, 900470 Constanta; ${ }^{4}$ Department of Nephrology, \\ ‘Sf. Apostol Andrei’ Emergency Clinical Hospital, 900591 Constanta, Romania
}

Received August 19,2021; Accepted September 20, 2021

DOI: $10.3892 /$ etm.2021.10959

\begin{abstract}
Acute kidney injury (AKI) is one of the most severe complications of SARS-CoV-2 infection. In a retrospective study, we aimed to describe the influence of COVID-19-related factors on the severity, outcome and timing of AKI in 268 patients admitted in two large COVID-19-designated university hospitals over a period of 6 months. In the univariate analysis, there was a significant relationship between KDIGO stage and the extension of COVID-19 pneumonia on computed tomography (CT), need for oxygen supplementation, serum levels of ferritin, interleukin-6, and procalcitonin, but none of these variables had a value for predicting KDIGO stage in multinomial regression. The odds of recovery of renal function were significantly diminished by d-dimer values. Lack of immunomodulatory treatment was found to be correlated with increased need for renal replacement therapy (RRT). Compared with AKI at admission, hospital-acquired AKI was predicted by the severity of lung damage on CT, evolved more frequently with incomplete recovery of renal function, and was significantly associated with antiviral therapy.
\end{abstract}

\section{Introduction}

Endemic SARS-CoV-2 infection has changed the life of both patients and doctors. Although a primary respiratory disease, involvement of other organs and systems has been described in severe cases $(1,2)$. Among these, COVID-19-associated acute kidney injury (AKI) represents an area of interest due to the

Correspondence to: Dr Cristiana David, Clinical Department No. 3, 'Carol Davila' University of Medicine and Pharmacy, 8 Eroii Sanitari Street, 050474 Bucharest, Romania

E-mail: cristianadavid@yahoo.com

Key words: SARS-CoV-2 infection, acute kidney injury, markers of inflammation, percentages of affected pulmonary parenchyma, renal outcome, timing of acute kidney injury high lethal potential of AKI and due to the lack of knowledge on the medium and long-term effects.

The reported incidence of COVID-19-associated AKI in different studies is very heterogeneous, varying between 2 and $23 \%$ (2-4), but most large meta-analyses describe an incidence between 10 and $15 \%$ of all COVID-19 patients $(1,5,6)$ and higher in subgroups of elderly and/or ICU-admitted patients. Reported consequences of the association between AKI and SARS-CoV-2 infection also vary in the literature, as there are studies recording the lack of negative impact on patient evolution (7) to studies proving increased death rates with increasing creatinine, KDIGO stage and/or need for renal replacement therapy (RRT) $(8,9)$.

In the present study, we aimed to describe the relationships between severity markers of SARS-CoV-2 infection and the evolutive characteristics of AKI in patients with moderate and severe forms of COVID-19 admitted to two university hospitals in Romania over a period of 6 months.

\section{Patients and methods}

A retrospective study was performed including adult patients with AKI and acute-on-chronic kidney disease (ACKD) admitted to two emergency university hospitals in Romania, 'Sf. Ioan' Emergency Clinical Hospital Bucharest and 'Sf. Apostol Andrei' Emergency Clinical Hospital Constanta between November 1, 2020 and April 30, 2021 (6 months). Both hospitals were designated for admission of SARS-CoV-2-infected patients. Moreover, being the only COVID-dedicated hospitals in the geographical area with emergency nephrology departments and the possibility to provide RRT 24/7, patients having both SARS-CoV-2 infection and renal involvement were preferentially orientated toward these medical facilities.

Patient data. Information for the study was retrieved from the electronic databases of the two hospitals. We collected demographic data (age, sex, comorbidities), laboratory findings related to SARS-CoV-2 infection [complete blood count, erythrocyte sedimentation rate (ESR), C-reactive protein (CRP), 
serum interleukin-6 (IL-6), procalcitonin (PCT), d-dimers, ferritin] or to kidney function [blood urea and creatinine $(\mathrm{Cr})]$. Peak values of these parameters were used in analysis except for lymphocyte count for which we used the lowest values during hospitalization. Severity of COVID-19 pneumonia was assessed by a radiologist in percentages of affected pulmonary parenchyma (PAPP) on chest computed tomography (CT). Need and types of oxygen supplementation were recorded. Data regarding treatment (antivirals, immunomodulators, RRT) and outcome (recovery of the renal function or death) were also analyzed in the study. Blood and urine tests were performed according to identical techniques and the results had the same reference range in both reporting hospitals.

$A K T$. Diagnosis of AKI was established according to KDIGO 2012 criteria (10): increase in serum $\mathrm{Cr} \geq 0.3 \mathrm{mg} / \mathrm{dl}$ within $48 \mathrm{~h}$ and/or increase of $>1.5$ times the previous values in the last 7 days. Although recorded, urine output was not a diagnostic criterion for inclusion. AKI was classified according to etiologic criteria (prerenal vs. intrinsic AKI) and also to timing criteria (AKI at admission vs. hospital-acquired AKI). Patients with previously diagnosed chronic kidney disease (CKD) were classified as acute-on-chronic-kidney disease (ACKD). Recovery of renal function was considered total when serum $\mathrm{Cr}$ levels returned to normal values (in AKI patients) or when it returned to baseline values before admission (in ACKD patients).

In order to evaluate the impact of AKI-COVID 19-association on patient outcome, we analyzed relationships between severity and timing of AKI and different parameters related to the severity of COVID-19.

Statement of ethical approval. Ethics Committees from both Hospitals were informed, and approvals for the study no. 4925/09.03.2021 ('Sf. Ioan' Hospital) and respectively no 30846/7.06.2021 ('Sf. Apostol Andrei' Hospital) were obtained. All patients read and signed written consent for inclusion in the study.

Statistical analysis. All the data was analyzed using IBM SPSS Statistics 25 (IBM, Corp.). Quantitative variables were tested for normal distribution using the Shapiro-Wilk Test and were expressed as averages with standard deviations or medians with interquartile ranges. Qualitative variables were expressed as counts or percentages. Quantitative variables were tested using Mann-Whitney U/Kruskal-Wallis H/One-way ANOVA tests, according to their distribution. Qualitative variables were tested using Pearson Chi-square tests/Fisher's exact tests. Logistic/Multinomial regression models were used to calculate odds ratios (OR) for possible risk factors in this study (significance of the models, goodness of fit, multicollinearity and linearity for quantitative variables were checked for the validity of the models).

\section{Results}

After excluding obstructive forms of AKI, 268 patients were included in the study (average age 72.28 years, 169 were men); 157 had AKI and 111 ACKD. Characteristics of the patients are documented in Table I. There was a high incidence of associated comorbidities, especially hypertension (79.1\%), coronary artery disease (58.2\%), chronic heart failure (56\%), and diabetes mellitus (42.5\%); 217 patients presented with prerenal AKI (80.97\%) and 51 patients had intrinsic AKI (19.03\%). RRT was necessary in 32 patients (11.94\%) (Table I).

According to KDIGO creatinine criteria, there were 81 patients $(30.22 \%)$ with stage $1,79(29.47 \%)$ with stage 2 and $108(40.29 \%)$ with stage 3 . Analyzing the relation between KDIGO stages and markers of COVID-19 severity (Table II), we found in the univariate analysis that CRP, ESR, fibrinogen and lymphocytes values were not significantly different between KDIGO stages, but patients with stage 3 had significantly higher PAPP (median, 75\%) and ferritin (median, 1,679 $\mathrm{ng} / \mathrm{ml}$ ) compared to the patients in stage 1 [affected lung (median, 50\%), ferritin (median, 1,014 ng/ml) $(\mathrm{P}<0.05)]$. Patients with stage 1 had significantly lower values of IL-6 (median, $64 \mathrm{pg} / \mathrm{ml}$ ) in comparison with patients in stage 2 (median, $157.4 \mathrm{pg} / \mathrm{ml}$ ) or patients with stage 3 (median, $310 \mathrm{pg} / \mathrm{ml})(\mathrm{P}<0.001)$. Patients with stage 1 were more frequently without the need of oxygen or needed supplementation on mask (40 or $40.5 \%$, respectively), while patients with stage 3 needed more frequent continuous positive airway pressure (CPAP) or invasive mechanical ventilation with endotracheal intubation (ETI) (51.3 or $54.8 \%$, respectively) $(\mathrm{P}<0.001)$. There was a gradual increase in PCT between AKI stages, patients with stage 1 having significantly lower values of PCT (median, $0.235 \mathrm{ng} / \mathrm{ml}$ ) in comparison to patients with stage 2 (median, $0.825 \mathrm{ng} / \mathrm{ml}$ ) or stage 3 (median, $2.39 \mathrm{ng} / \mathrm{ml}$ ), the differences between stages 2 and 3 being also significant $(\mathrm{P}<0.001)$. In addition, patients in stage $3 \mathrm{KDIGO}$ were more frequently admitted to the ICU than those in stage 1 (55 vs. $14.4 \%$; $\mathrm{P}<0.001$ ). Nevertheless, a multinomial regression model using ferritin, PAPP, IL-6 and PCT was tested, but the model was not significant $(\mathrm{P}=0.068)$, therefore no variables could be used to predict the KDIGO stages (Table III).

In total, 135 patients $(50.37 \%)$ died during hospitalization. In the survivors, full recovery of renal function after AKI episode was registered in 73 patients, and partial recovery in 52 patients; 8 patients became hemodialysis (HD)-dependent during hospitalization. Analyzing the relation between recovery of renal function and markers of COVID-19 severity (Table III), we found that PCT, d-dimer levels and AKI timing were significantly different between renal function recovery groups $(\mathrm{P}<0.05)$; as such, patients with no recovery of renal function (HD-dependence) had significantly higher values of PCT (median, $7.43 \mathrm{ng} / \mathrm{ml}$ ) and d-dimer levels (median, $7.98 \mu \mathrm{g} / \mathrm{ml}$ ) in comparison to patients with total recovery [PCT (median, $0.26 \mathrm{ng} / \mathrm{ml}$ ), d-dimer (median, $1.99 \mu \mathrm{g} / \mathrm{ml}$ )] or patients with partial recovery [PCT (median, $0.33 \mathrm{ng} / \mathrm{ml}$ ), d-dimer (median, $2.03 \mu \mathrm{g} / \mathrm{ml})(\mathrm{P}<0.05)]$. Patients with partial renal function recovery were significantly associated with hospital-acquired AKI (53.8\% vs. 35.5\%; P=0.023) (Table III). In a multinomial regression model (with total recovery as a reference) (Table IV), only d-dimer levels had a significant prediction $(\mathrm{P}=0.011)$, showing that an increase in $\mathrm{d}$-dimer levels with 1 unit raises the odds of having no recovery of renal function by 1.128 times (95\% CI, 1.028-1.237).

Antiviral therapy including remdesivir, favipiravir, remdesivir + favipiravir or lopinavir/ritonavir was 
Table I. Characteristics of the patients $(\mathrm{N}=268)$ included in the study.

\begin{tabular}{|c|c|c|c|}
\hline Variables & $\mathrm{n}(\%)$ & Laboratory tests (n) & $\begin{array}{c}\text { Peak values } \\
\text { (average) }\end{array}$ \\
\hline Male sex & $169(63.1)$ & Urea $(\mathrm{mg} / \mathrm{dl})(268)$ & 164.5 \\
\hline Prerenal AKI & $217(80.9)$ & Creatinine (mg/dl) (268) & 3.41 \\
\hline Intrinsic AKI & $51(19.0)$ & & \\
\hline AKI & $157(58.6)$ & Procalcitonin (ng/ml) (217) & 10.13 \\
\hline ACKD & $111(41.4)$ & & \\
\hline Diabetes mellitus & $114(42.5)$ & CRP (mg/l) (264) & 134.2 \\
\hline Neoplastic disease & $47(17.5)$ & IL-6 (pg/ml) (199) & $5,154.76$ \\
\hline Chronic liver disease & $57(21.3)$ & $\operatorname{ESR}(\mathrm{mm} / \mathrm{h})(199)$ & 69.43 \\
\hline Heart failure & $150(56)$ & Ferritin (ng/ml) (234) & $3,292.97$ \\
\hline Atherosclerosis & $123(45.9)$ & Fibrinogen (mg/dl) (258) & 637.29 \\
\hline Hypertension & $212(79.1)$ & D-dimer $(\mu \mathrm{g} / \mathrm{ml})(239)$ & 5.26 \\
\hline Coronary artery disease & $156(58.2)$ & Lymphocyte count (/mmc) (268) & 997.94 \\
\hline Oxygen therapy & & Evolution during hospitalization & $\mathrm{n}(\%)$ \\
\hline Mask & $149(55.5)$ & Sepsis & $150(56.0)$ \\
\hline CPAP & $63(23.5)$ & RRT & $32(11.9)$ \\
\hline ETI & $73(26.2)$ & Death & $135(50.3)$ \\
\hline \multicolumn{4}{|l|}{ Chest CT } \\
\hline Quantified lesions & $140(52.3)$ & & \\
\hline No valid data & $128(47.7)$ & & \\
\hline \multicolumn{4}{|l|}{ Antivirals } \\
\hline Remdesivir & 105 (39.2) & & \\
\hline Favipiravir & $42(15.7)$ & & \\
\hline Remdesivir + favipiravir & $4(1.5)$ & & \\
\hline Lopinavir/ritonavir & $9(3.3)$ & & \\
\hline \multicolumn{4}{|l|}{ Immunomodulators } \\
\hline Tocilizumab & $22(8.2)$ & & \\
\hline Anakinra & $90(33.6)$ & & \\
\hline Tocilizumab + anakinra & $13(4.8)$ & & \\
\hline
\end{tabular}

AKI, acute kidney injury; ACKD, acute-on-chronic kidney disease; CPAP, continuous positive airway pressure; ETI, endotracheal intubation; CT, computer tomography; CRP, C-reactive protein; IL-6, interleukin 6; ESR, erythrocyte sedimentation rate; RRT, renal replacement therapy.

recommended in 171 patients, and immunomodulators (anakinra, tocilizumab or both) in 136 patients (Table I). Statistical analysis (Table V) showed that patients treated with antivirals developed AKI during hospitalization significantly more frequently than those without antivirals (73.5\% vs. $59.5 \%$; $\mathrm{P}=0.027$ ), but no significant relationship was found between the presence of antiviral therapy and KDIGO stages or dialysis requirement $(\mathrm{P}>0.05)$. Patients without immunomodulatory treatment had a significantly higher need for RRT in comparison with those receiving this treatment (53\% vs. $34.4 \%$; $\mathrm{P}=0.048)$ (Table V).

In 185 patients $(69.02 \%)$, AKI was diagnosed at admission; 83 patients $(30.97 \%)$ developed AKI episode during hospitalization. There was no significant difference between the two groups regarding age, comorbidities frequencies, levels of PCT, IL-6, ferritin and d-dimers, nor regarding etiology of AKI or period between COVID-19 onset symptoms and hospital admission $(\mathrm{P}>0.05)$. Instead, we found that patients with AKI at admission had more frequently history of previous CKD (79.3\% vs. $20.7 \%$; $\mathrm{P}=0.003)$ and also a significant higher need for RRT ( $90.6 \%$ vs. $66.1 \%$; $\mathrm{P}=0.004)$. Patients with hospital-acquired AKI had significantly higher PAPP on CT-scan (median, $75 \%$ vs. 50\%) $(\mathrm{P}<0.001)$ (Table VI). Using a logistic regression model, it was shown that the values of PAPP on CT-scan had a significant prediction value $(\mathrm{P}<0.001)$ (Table VI); each increase of a percentage unit in affected lung increased the odds of having AKI during hospitalization by 1.027 times (95\% CI, 1.012-1.041).

\section{Discussion}

General data. In this retrospective analysis, we aimed to identify the influence of COVID-19-related factors on the severity, timing and outcome of AKI. Out cohort consisted of 268 adult SARS-CoV-2-infected patients with AKI, either present from the moment of admission or developed during hospitalization. The literature discusses the possible risk of more severe forms of the disease if the virus is inoculated after vaccination, but 
Table II. Comparison between COVID-19 severity markers and AKI KDIGO stage.

\begin{tabular}{|c|c|c|c|c|}
\hline Parameters & KDIGO 1 & KDIGO 2 & KDIGO 3 & P-value \\
\hline No. of patients (\%) & $81(30.22 \%)$ & $79(29.47 \%)$ & $108(40.29 \%)$ & \\
\hline PAPP-CT (\%) & $50(25-75)$ & $50(30-75)$ & $75(40-80)$ & $0.044^{a}$ \\
\hline Ferritin $(\mathrm{ng} / \mathrm{ml})$ & $1,014(505.8-1937.5)$ & $1,317(711-2,716)$ & $1,679(1,152-4,355)$ & $<0.001^{\mathrm{a}}$ \\
\hline IL-6 (pg/ml) & $64(13.55-298.45)$ & $157.4(50.3-1120.8)$ & $310(67.7-1,673)$ & $<0.001^{\mathrm{a}}$ \\
\hline CRP (mg/l) & $105.5(59.8-154.4)$ & $124.9(68.2-225.9)$ & $102.1(27.5-215.2)$ & $0.192^{\mathrm{a}}$ \\
\hline $\mathrm{ESR}(\mathrm{mm} / 1 \mathrm{~h})$ & $58.5(42.2-80)$ & $70(52-100)$ & $77(51-101)$ & $0.077^{\mathrm{a}}$ \\
\hline Fibrinogen (mg/dl) & $635.38 \pm 173.27$ & $626.68 \pm 194.06$ & $646.51 \pm 212.54$ & $0.796^{\mathrm{b}}$ \\
\hline Lymphocytes (/mmc) & $630(380-945)$ & $610(400-960)$ & $520(352.5-765)$ & $0.180^{\mathrm{a}}$ \\
\hline PCT (ng/ml) & $0.235(0.122-0.6)$ & $0.825(0.26-3.07)$ & $2.39(0.47-11.25)$ & $<0.001^{\mathrm{a}}$ \\
\hline \multicolumn{5}{|l|}{ Oxygen therapy } \\
\hline Absent & $18(40 \%)$ & $9(20 \%)$ & $18(40 \%)$ & $<0.001^{\mathrm{c}}$ \\
\hline Mask & $45(40.5 \%)$ & $36(32.4 \%)$ & $30(27 \%)$ & \\
\hline CPAP & $10(25.6 \%)$ & $9(23.1 \%)$ & $20(51.3 \%)$ & \\
\hline ETI & $8(11 \%)$ & $25(34.2 \%)$ & $40(54.8 \%)$ & \\
\hline \multicolumn{5}{|l|}{ ICU admission } \\
\hline Absent & $65(41.4 \%)$ & $45(28.7 \%)$ & $47(29.9 \%)$ & $<0.001^{\mathrm{c}}$ \\
\hline Present & $16(14.4 \%)$ & $34(30.6 \%)$ & $61(55 \%)$ & \\
\hline
\end{tabular}

Data are expressed as average $\pm \mathrm{SD}$, median $(\mathrm{IQR})$ or n (\%). ${ }^{\mathrm{a}}$ Kruskal Wallis-H test, ${ }^{\mathrm{b} O n e-w a y ~ A N O V A}$ test, ${ }^{\mathrm{c}}$ Fisher's exact test. Significant P-values are indicated in bold print. AKI, acute kidney injury; PAPP-CT, pulmonary parenchyma on chest computer tomography; IL-6, interleukin 6; CRP, C-reactive protein; ESR, erythrocyte sedimentation rate; PCT, procalcitonin; CPAP, continuous positive airway pressure; ETI, endotracheal intubation; RRT, renal replacement therapy.

Table III. Comparison between COVID-19 severity markers and recovery of renal function.

\begin{tabular}{|c|c|c|c|c|}
\hline Parameter & Total recovery & Partial recovery & Dependence of HD & P-value \\
\hline No. of patients & 73 & 52 & 8 & \\
\hline PAPP-CT (\%) & $47.5(25-62.5)$ & $30(15-67.5)$ & $45(15-60)$ & $0.646^{\mathrm{a}}$ \\
\hline Ferritin (ng/ml) & $1,133.5(610.1-2,015.7)$ & $935.2(557.2-1,549.2)$ & $1,517.5(1,042-3,704)$ & $0.225^{\mathrm{a}}$ \\
\hline IL-6 (pg/ml) & $62.4(20.47-186.4)$ & $78(25.47-233.15)$ & $237.2(46.45-881.8)$ & $0.352^{\mathrm{a}}$ \\
\hline $\mathrm{CRP}(\mathrm{mg} / \mathrm{l})$ & 97.3(58.4-144.26) & $78.48(15.05-164.24)$ & $61.36(16.1-140.43)$ & $0.344^{\mathrm{a}}$ \\
\hline $\operatorname{ESR}(\mathrm{mm} / 1 \mathrm{~h})$ & $63(42.75-89.25)$ & $69(48.75-94.5)$ & $88(66.25-106.5)$ & $0.096^{\mathrm{a}}$ \\
\hline Fibrinogen $(\mathrm{mg} / \mathrm{dl})$ & $638.82 \pm 181.43$ & $624 \pm 149.36$ & $664.14 \pm 213.87$ & $0.714^{\mathrm{b}}$ \\
\hline Lymphocytes (/mmc) & $695(477.5-972.5)$ & $600(420-990)$ & $695(352.5-1,087.5)$ & $0.738^{\mathrm{a}}$ \\
\hline $\mathrm{D}$-dimer $(\mu \mathrm{g} / \mathrm{ml})$ & $1.99(0.93-3.495)$ & $2.03(1.16-7.18)$ & $7.98(3.57-11.31)$ & $0.002^{\mathrm{a}}$ \\
\hline PCT (ng/ml) & $0.26(0.1-0.835)$ & $0.33(0.14-1.42)$ & $7.43(0.84-15.66)$ & $\mathbf{0 . 0 0 3}{ }^{\mathrm{a}}$ \\
\hline \multicolumn{5}{|l|}{ AKI timing } \\
\hline At admission & $61(57 \%)$ & $38(35.5 \%)$ & $8(7.5 \%)$ & \multirow[t]{2}{*}{$0.023^{c}$} \\
\hline Hospital acquired & $12(46.2 \%)$ & $14(53.8 \%)$ & $0(0 \%)$ & \\
\hline \multicolumn{5}{|l|}{ Antiviral therapy } \\
\hline Absent & $27(55.1 \%)$ & $20(40.8 \%)$ & $2(4.1 \%)$ & \multirow[t]{2}{*}{$0.758^{c}$} \\
\hline Present & $46(54.8 \%)$ & $32(38.1 \%)$ & $6(7.1 \%)$ & \\
\hline \multicolumn{5}{|l|}{ Immunomodulators } \\
\hline Absent & $34(41.5 \%)$ & $38(46.3 \%)$ & $10(12.2 \%)$ & \multirow[t]{2}{*}{$0.137^{\mathrm{c}}$} \\
\hline Present & $44(57.1 \%)$ & $27(35.1 \%)$ & $6(7.8 \%)$ & \\
\hline
\end{tabular}


Table IV. Multinomial regression for renal function recovery of possible risk factors.

\begin{tabular}{llcl}
\hline Parameter & \multicolumn{1}{c}{ Prediction } & OR $(95 \% \mathrm{CI})$ & $(\mathrm{P}$-value $)$ \\
\hline D-dimer & Partial vs. total & $1.047(0.974-1.127)$ & $(\mathrm{P}=0.214)$ \\
& None vs. total & $1.128(1.028-1.237)$ & $(\mathrm{P}=\mathbf{0 . 0 1 1})$ \\
PCT & Partial vs. total & $0.975(0.937-1.015)$ & $(\mathrm{P}=0.224)$ \\
& None vs. total & $1.006(0.976-1.038)$ & $(\mathrm{P}=0.680)$ \\
\multirow{4}{*}{ AKI timing } & Partial vs. total & $2.728(0.921-8.081)$ & $(\mathrm{P}=0.070)$ \\
& None vs. total & $2.976(0.595-14.898)$ & $(\mathrm{P}=0.184)$ \\
\hline
\end{tabular}

PCT, procalcitonin; AKI, acute kidney injury; OR, odds ratio; CI, confidence interval. Significant P-values are indicated in bold print.

none of the patients enrolled in the study had been vaccinated prior to hospitalization (11).

Most of our patients were elderly (average 72.28 years), male sex and carried a significant burden of comorbidities, data similar with other reports $(6,12,13)$. A total of $69.77 \%$ had AKI in KDIGO stage 2 and 3; AKI in the context of COVID-19 has been shown to be more severe compared to other etiologies (14).

Severity of COVID-19 and KDIGO stages. We found in the univariate analysis a direct relationship between KDIGO stage and severity of some of the markers of COVID-19 including serum values of interleukin (IL)-6, procalcitonin (PCT), ferritin, d-dimers, pulmonary parenchyma (PAPP) on chest computer tomography (CT). However, in the logistic multinomial regression, the KDIGO stage could not be predicted by any of the listed markers.

Several studies have reported that inflammatory markers in COVID-19 are predictive both for the occurrence and severity of AKI (15-18). Severe lung injury is associated with the overproduction of pro-inflammatory cytokines which can increase the risk of AKI (19), as was documented, at least experimentally, even before the COVID-19 pandemic (20). Nevertheless, in COVID-19, literature findings during this period also provide reports concluding that markers of inflammation are rather predictive for the severity of lung damage than for the severity of AKI (21) and reports finding no relationship between these markers and severity of kidney injury exist (22). We can speculate that some factors did influence our results. One factor may be the assessment of the extent of COVID-19 pneumonia on CT. Quantification of lesions was performed in 140 patients; a degree of inter-observer variability may have been present in interpreting the results; chest CT-scan was not always repeated during hospitalization if patient treatment was not changed (i.e. if the patient had maximum available treatment, no benefits would have been obtained with a new CT). A similar situation was noted regarding serum inflammatory markers; these variables were not analyzed in all patients for reason of availability (Table I). In addition, we used in the statistical analysis the maximum values which could have been influenced by the treatment with immunomodulators; inflammatory markers may be elevated also in the presence of CKD or different comorbidities (23-25). Yet, the most valid explanation for the lack of correlation between inflammatory markers and KDIGO stages in multivariate analysis, although highly significant in the univariate analysis, was that peak levels of these markers were not simultaneously noted in the same individual during hospitalization (i.e. PCT increases later when sepsis is superimposed; IL-6 increases at a maximum level after IL-6-antagonists).

Severity of COVID-19 and recovery of renal function. In the multivariate analysis we found that, among markers of COVID-19 severity, only d-dimers were predictive for permanent loss of kidney function after COVID-19-associated AKI. This result, in line with other reports (26), suggests that intrarenal microthrombosis may be an important factor in COVID-19-associated kidney injury. Moreover, patients with hospital-acquired AKI, although most having previous normal kidney function, were discharged with lower recovery of kidney function in comparison with AKI-at-onset cases which included many ACKD cases. Nevertheless, our explanation is just speculative because the occurrence of renal capillary thrombosis seems to be more complex involving also markers of inflammation which were not found to influence renal recovery $(27,28)$.

Impact of COVID-19-specific treatment and severity of AKI. We revealed a significant association between antiviral therapy and the risk for developing AKI during hospitalization; we can only suspect nephrotoxicity of the antivirals and there are few reports in this regard (29-31) although the vast majority of data pleads for their safety.

Regarding our finding that patients without immunomodulatory treatment had significantly higher need for RRT, other reports pointed out favorable clinical response after anakinra administration in the cytokine storm state (32) and that early use of immunomodulatory drugs may protect from severe complications (33). It is difficult in a retrospective analysis to define the exact timing of immunomodulator initiation.

AKI timing. Almost two thirds of our patients presented with AKI at the moment of admission, most of them being known with a history of CKD. Numerous studies report that AKI at admission is frequent in COVID-19 (34-36), but, regarding prevalence of previous CKD, some authors found it higher in AKI at admission $(34,37)$ similar to our study, whereas others in hospital-developed AKI (38). This difference was probably due to the modality of admission in the hospital, availability and extension of ICU departments in different hospitals, type of treatment available and recommended by evolving guidelines in the last year of the pandemic.

We found no differences between AKI at admission and hospital-acquired AKI regarding age or comorbid burden. Most authors report age and multiple comorbidities as predictive factors for AKI at admission $(34,37)$ and few found advanced age to be associated with hospital-acquired AKI (38).

Regarding the influence of COVID-19 severity markers on the timing of AKI, we found in multinomial regression a significant relationship only between PAPP and hospital-acquired AKI. While several studies report an association between inflammatory markers in COVID-19 and severity of AKI, 
Table V. Parameters influenced by COVID-19-specific therapy.

\begin{tabular}{lcc}
\hline Parameter & Antiviral - & Antiviral + \\
\hline AKI KDIGO stage & & \\
KDIGO 1 & $29(35.8 \%)$ & $52(64.2 \%)$ \\
KDIGO 2 & $25(31.6 \%)$ & $54(68.4 \%)$ \\
KDIGO 3 & $43(39.8 \%)$ & $65(60.2 \%)$ \\
AKI timing & & \\
At admission & $75(40.5 \%)$ & $110(59.5 \%)$ \\
Hospital acquired & $22(26.5 \%)$ & $61(73.5 \%)$ \\
RRT need & $84(35.6 \%)$ & $152(64.4 \%)$ \\
Absent & $13(40.6 \%)$ & $19(59.4 \%)$ \\
Present & Immunomodulatory & $125(53 \%)$ \\
RRT need & $111(47 \%)$ & $11(34.4 \%)$ \\
Absent & $21(65.6 \%)$ & $\mathbf{0 . 0 2 7}$ \\
Present & & \\
\end{tabular}

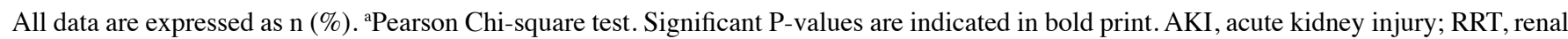
replacement therapy.

Table VI. Parameters with statistical power regarding AKI timing.

\begin{tabular}{|c|c|c|c|}
\hline Parameter & At admission & During hospitalization & P-value \\
\hline Total no $(\%)$ of patients & $185(69.02 \%)$ & $83(30.97 \%)$ & \\
\hline \multicolumn{4}{|l|}{ AKI type } \\
\hline ACKD & $88(79.3 \%)$ & $23(20.7 \%)$ & $0.003^{b}$ \\
\hline AKI & $97(61.8 \%)$ & $60(38.2 \%)$ & \\
\hline RRT need & $156(66.1 \%)-/ 29(90.6 \%)+$ & $80(33.9 \%)-/ 3(9.4 \%)+$ & $0.004^{b}$ \\
\hline PAPP-CT (\%) & $50(25-75)$ & $75(47.5-80)$ & $<0.001^{\mathrm{a}}$ \\
\hline
\end{tabular}

Data are expressed as median (IQR) or n (\%). ${ }^{\mathrm{a}}$ Mann-Whitney U test, ${ }^{\mathrm{b}}$ Fisher's exact test. Significant P-values are indicated in bold print. AKI, acute kidney injury; ACKD, acute-on-chronic kidney disease; RRT, renal replacement therapy; PAPP-CT, pulmonary parenchyma on chest computer tomography.

few authors have addressed the subject of timing and they also found no differences between the two types of AKI (34). Nevertheless, and this is one of the limits of our present study, we did not correlate COVID-19 severity markers with presence, type and timing of treatment applied as the treatment may modify levels of different variables; also, in the analysis, we used the peak values of inflammatory markers and these levels may be modified by both treatment or other complications. The significance of our finding, that PAPP could predict onset of AKI during hospitalization, may partially be related to the increased need of invasive supplementation of oxygen in patients with severe COVID-19 pneumonia, as it is known that mechanical ventilation increases the risk of AKI secondary to selective renal vasoconstriction (39). Moreover, in the light of this finding, we can also speculate that prophylactic approach in this matter in our hospitals-ETI and invasive ventilation before occurrence of severe respiratory failure with hemodynamic instability-had both advantages and disadvantages and may also explain the increased number of prerenal AKI among hospital-acquired AKI.

The last observation is that cases with hospital-acquired AKI had in the same time, in comparison with AKI at admission, both a lower need for RRT, but surprisingly less complete recovery of renal function, although there were more AKI than ACKD cases in this subgroup. These data may be explained by the fact that a lot of AKI cases at admission were most likely due to prerenal rapidly reversible causes (fever, vomiting, diarrhea), and also by the fact that patients with previously CKD needed prompt RRT which allowed, in the majority of cases, full recovery of renal function (ie reversal to the same creatinine level as before COVID-19). In the same time, patients who developed AKI during hospitalization were those in who COVID-19 aggravated progressively with superimposed both prerenal and intrarenal insults. Prerenal injury acquired/developed during hospitalization was in the context of hemodynamic renal alteration secondary to causes 
less responsive to treatment (sepsis, mechanical ventilation, aggravation of previous cardiac failure).

In conclusion, the present study brings additional information in the field of COVID-19-associated AKI. Retrospectively analyzing patients hospitalized with COVID-19 and AKI, we did not find a correlation, in the multinomial analysis, between the severity markers of COVID-19 and AKI KDIGO stage. Decreased odds for recovery of kidney function were associated with peak levels of d-dimers. Lack of immunomodulatory treatment was found to be correlated with increased need for RRT. Compared with AKI at admission, hospital-acquired AKI was better predicted by severity of lung damage on $\mathrm{CT}$, evolved more frequently with incomplete recovery of renal function, and was significantly associated with antiviral therapy.

\section{Acknowledgements}

Not applicable.

\section{Funding}

No funding was received.

\section{Availability of data and materials}

The datasets used and/or analyzed during the current study are available from the corresponding author on reasonable request.

\section{Authors' contributions}

DR, LAT, CD and IAV conceived the study. DR, CD and SDO drafted the manuscript. CB, SDO, EC, ES, AC, LFF, CP and MCN collected, analyzed and interpreted the data. All authors read and approved the final manuscript for publication.

\section{Ethics approval and consent to participate}

The study protocol was approved by the Ethics Committees of the two hospitals: 'Sf. Ioan' Emergency Clinical Hospital, Bucharest, Romania (no. 4925/09.03.2021) and 'Sf. Apostol Andrei' Emergency Clinical Hospital, Constanta, Romania (no. 30846/7.06.2021). All patients read and signed the written informed consent. The study was performed in accordance with Good Clinical Practice and the ethical standards of the Declaration of Helsinki (revised 2014).

\section{Patient consent for publication}

Not applicable.

\section{Competing interests}

The authors declare that they have no competing interests.

\section{References}

1. Fu L, Wang B, Yuan T, Chen X, Ao Y, Fitzpatrick T, Li P, Zhou Y, Lin YF, Duan Q, et al: Clinical characteristics of coronavirus disease 2019 (COVID-19) in China: A systematic review and meta-analysis. J Infect 80: 656-665, 2020.
2. Docea AO, Tsatsakis A, Albulescu D, Cristea O, Zlatian O, Vinceti M, Moschos SA, Tsoukalas D, Goumenou M, Drakoulis N, et al: A new threat from an old enemy: Re-emergence of coronavirus (review). Int J Mol Med 45: 1631-1643, 2020.

3. Huang C, Wang Y, Li X, Ren L, Zhao J, Hu Y, Zhang L, Fan G, Xu J, Gu X, et al: Clinical features of patients infected with 2019 novel coronavirus in Wuhan, China. Lancet 395: 497-506, 2020.

4. Wang D, Hu B, Hu C, Zhu F, Liu X, Zhang J, Wang B, Xiang H, Cheng Z, Xiong Y, et al: Clinical characteristics of 138 hospitalized patients with 2019 novel coronavirus-infected pneumonia in Wuhan, China. JAMA 323: 1061-1069, 2020.

5. Lin L, Wang X, Ren J, Sun Y, Yu R, Li K, Zheng L and Yang J: Risk factors and prognosis for COVID-19-induced acute kidney injury: A meta-analysis. BMJ Open 10: e042573, 2020.

6. Fabrizi F, Alfieri CM, Cerutti R, Lunghi G and Messa P: COVID-19 and acute kidney injury: A systematic review and meta-analysis. Pathogens 9: 1052, 2020.

7. Wang L, Li X, Chen H, Yan S, Li D, Li Y and Gong Z: Coronavirus disease 19 infection does not result in acute kidney injury: An analysis of 116 hospitalized patients from Wuhan, China. Am J Nephrol 51: 343-348, 2020.

8. Cheng Y, Luo R, Wang K, Zhang M, Wang Z, Dong L, Li J, Yao Y, Ge S and Xu G: Kidney disease is associated with in-hospital death of patients with COVID-19. Kidney Int 97: 829-838, 2020.

9. Paek JH, Kim Y, Park WY, Jin K, Hyun M, Lee JY, Kim HA, Kwon YS, Park JS and Han S: Severe acute kidney injury in COVID-19 patients is associated with in-hospital mortality. PLoS One 15: e243528, 2020.

10. Kidney Disease: Improving global outcomes (KDIGO). Acute kidney injury work group: KDIGO clinical practice guideline for acute kidney injury. Kidney Int Suppl 2: 1-138, 2012.

11. Calina D, Docea AO,Petrakis D,Egorov AM, Ishmukhametov AA, Gabibov AG, Shtilman MI, Kostoff R, Carvalho F, Vinceti M, et al: Towards effective COVID-19 vaccines: Updates, perspectives and challenges (review). Int J Mol Med 46: 3-16, 2020.

12. Kolhe NV, Fluck RJ, Selby NM and Taal MW: Acute kidney injury associated with COVID-19: A retrospective cohort study. PLoS Med 17: e1003406, 2020.

13. Wang B, Li R, Lu Z and Huang Y: Does comorbidity increase the risk of patients with Covid-19: Evidence from meta-analysis. Aging (Albany NY) 12: 6049-6057, 2020.

14. Moledina DG, Simonov M, Yamamoto Y, Alausa J, Arora T, Biswas A, Cantley LG, Ghazi L, Greenberg JH, Hinchcliff M, et al: The association of COVID-19 with acute kidney injury independent of severity of illness: A multicenter cohort study. Am J Kidney Dis 77: 490-499.e1, 2021.

15. Mohamed MMB, Lukitsch I, Torres-Ortiz AE, Walker JB, Varghese V, Hernandez-Arroyo CF, Alqudsi M, LeDoux JR and Velez JCQ: Acute kidney injury associated with coronavirus disease 2019 in Urban New Orleans. Kidney360 1: 614-622, 2020.

16. Xia P, Wen Y, Duan Y, Su H, Cao W, Xiao M, Ma J, Zhou Y, Chen G, Jiang W, et al: Clinicopathological features and outcomes of acute kidney injury in critically ill COVID-19 with prolonged disease course: A retrospective cohort. J Am Soc Nephrol 31: 2205-2221, 2020.

17. Phillips T, Stammers M, Leggatt G, Bonfield B, Fraser S, Armstrong K and Veigheyet K: Acute kidney injury in COVID-19: Identification of risk factors and potential biomarkers of disease in a large UK cohort. Nephrology 26: 420-431, 2021.

18. Aggarwal S, Garcia-Telles N, Aggarwal G, Lavie C, Lippi G and Henry BM: Clinical features, laboratory characteristics, and outcomes of patients hospitalized with coronavirus disease 2019 (COVID-19): Early report from the United States. Diagnosis (Berl) 7: 91-96, 2020.

19. Chen J, Wang W, Tang Y, Huang XR, Yu X and Lan HY: Inflammatory stress in SARS-COV-2 associated acute kidney injury. Int J Biol Sci 17: 1497-1506, 2021.

20. Nechemia-Arbely Y, Barkan D, Pizov G, Shriki A, Rose-John S, Galun E and Axelrod JH: IL-6/IL-6R axis plays a critical role in acute kidney injury. J Am Soc Nephrol 19: 1106-1115, 2008.

21. Joseph A, Zafrani L, Mabrouki A, Azoulay E and Darmon M: Acute kidney injury in patients with SARS-CoV-2 infection. Ann Intensive Care 10: 117, 2020.

22. Xiang HX, Fei J, Xiang Y, Xu Z, Zheng L, Li XY, Fu L and Zhao H: Renal dysfunction and prognosis of COVID-19 patients: A hospital-based retrospective cohort study. BMC Infect Dis 21: 158,2021

23. Su H, Lei CT and Zhang C: Interleukin-6 signaling pathway and its role in kidney disease: An update. Front Immunol 8: 405 , 2017. 
24. Grace E and Turner RM: Use of procalcitonin in patients with various degrees of chronic kidney disease including renal replacement therapy. Clin Infect Dis 59: 1761-1767, 2014.

25. Abbasi A, Corpeleijn E, Postmus D, Gansevoort RT, de Jong PE, Gans RO, Struck J, Hillege HL, Stolk RP, Navis G and Bakker SJ Plasma procalcitonin is associated with obesity, insulin resistance, and the metabolic syndrome. J Clin Endocrinol Metab 95: E26-E31, 2010.

26. Gupta S, Coca SG, Chan L, Melamed ML, Brenner SK, Hayek SS Sutherland A, Puri S, Srivastava A, Leonberg-Yoo A, et al: AKI treated with renal replacement therapy in critically ill patients with COVID-19. J Am Soc Nephrol 32: 161-176, 2021.

27. Chen X, Yu C, Jing H, Wang C, Zhao X, Zhang J, Zhang S, Liu H, Xie R and Shi J: COVID-19 associated thromboinflammation of renal capillary: Potential mechanisms and treatment Am J Transl Res 12: 7640-7656, 2020.

28. Ng JH, Bijol V, Sparks MA, Sise ME, Izzedine H and Jhaveri KD: Pathophysiology and pathology of acute kidney injury in patients with COVID-19. Adv Chronic Kidney Dis 27: 365-376, 2020.

29. Beigel JH, Tomashek KM, Dodd LE, Mehta AK, Zingman BS, Kalil AC, Hohmann E, Chu HY, Luetkemeyer A, Kline S, et al: Remdesivir for the treatment of Covid-19-final report. N Engl J Med 383: 1813-1826, 2020.

30. Goldman JD, Lye DCB, Hui DS, Marks KM, Bruno R, Montejano R, Spinner CD, Galli M, Ahn MY, Nahass RG, et al: Remdesivir for 5 or 10 days in patients with severe Covid-19. N Engl J Med 383: 1827-1837, 2020.

31. Nasa P, Shrivastava P, Kulkarni A, Vijayan L and Singh A. Favipiravir induced nephrotoxicity in two patients of COVID-19. J Assoc Physicians India 69: 90, 2021.

32. Nemchand P, Tahir H, Mediwake R and Lee J: Cytokine storm and use of anakinra in a patient with COVID-19. BMJ Case Rep 13: e237525, 2020.
33. Langer-Gould A, Smith JB, Gonzales EG, Castillo RD, Figueroa JG, Ramanathan A, Li BH and Gould MK: Early identification of COVID-19 cytokine storm and treatment with anakinra or tocilizumab. Int J Infect Dis 99: 291-297, 2020.

34. Martínez-Rueda AJ, Álvarez RD, Méndez-Pérez RA, Fernández-Camargo DA, Gaytan-Arocha JE, Berman-Parks N, Flores-Camargo A, Comunidad-Bonilla RA, Mejia-Vilet JM, Arvizu-Hernandez M, et al: Community- and hospital-acquired acute kidney injury in COVID-19: Different phenotypes and dismal prognosis. Blood Purif 50: 931-941, 2021.

35. Pelayo J, Lo KB, Bhargav R, Gul F, Peterson E, DeJoy III R, Salacup GF, Albano J, Gopalakrishnan A, Azmaiparashvili Z, et al: Clinical characteristics and outcomes of community- and hospital-acquired acute kidney injury with COVID-19 in a US inner city hospital system. Cardiorenal Med 10: 223-231, 2020.

36. Portolés J, Marques M, López-Sánchez P, de Valdenebro M, Muñez E, Serrano ML, Malo R, García E and Cuervas V: Chronic kidney disease and acute kidney injury in the COVID-19 Spanish outbreak. Nephrol Dial Transplant 35: 1353-1362, 2020.

37. Tarragón B, Valdenebro M, Serrano ML, Maroto A, Llópez-Carratalá MR, Ramos A, Rubio E, Huerta A, Marques M and Portolés J: Acute kidney failure in patients admitted due to COVID-19. Nefrología (Engl Ed) 41: 34-40, 2021 (In English, Spanish).

38. Peng S, Wang HY, Sun X, Li P, Ye Z, Li Q, Wang J, Shi X, Liu L, Yao Y, et al: Early versus late acute kidney injury among patients with COVID-19-a multicenter study from Wuhan, China. Nephrol Dial Transplant 35: 2095-2102, 2020.

39. van den Akker JP, Egal $M$ and Groeneveld AB: Invasive mechanical ventilation as a risk factor for acute kidney injury in the critically ill: A systematic review and meta-analysis. Crit Care 17: R98, 2013. 